\title{
Observations sur la kératoconjonctivite infectieuse du bovin en Côte d'Ivoire $(*)$
}

\author{
2. Etude sur le rôle vecteur des lépidoptères ophtalmotropes
}

par J. NICOLET $(* *)$ et W. BUTTIKER $(* * *)$

\begin{abstract}
RESUME
Les auteurs ont tenté dans cette étude informative de cerner le problème du rôle jouć par les lépidoptères ophtalmotropes dans la transmission de la kératoconjonctivite du bovin. L'isolement sur le proboscis de certains germes, notamment Moraxella nonliquefaciens, dans l'intestin et les excréments de Neisseria catarrhalis var, haemolytica, de Klebsiella aerogenes et d'Aeromonas sp. laissent supposer que les papillons peuvent transmettre de telles bactéries d'un animal à l'autre. Aucun mycoplasme n'a pu être isolé du proboscis, de l'intestin ou des excréments de onze papillons examinés.
\end{abstract}

\section{INTRODUCTION}

Les lépidoptères ophtalmotropes sont des papillons nocturnes appartenant essentiellement aux noctuidés, pyralidés et géométridés. Ils se nourrissent principalement de sécrétion lacrymale de mammifères, en se posant près de la paupière de l'hôte et atteignant la conjonctive à l'aide du proboscis (photo).

Les aspects concernant la biologie, la répartition en Afrique et en Asie, la nutrition etc., de ces papillons ophtalmotropes ont fait l'objet de nombreuses publications, citées dans le travail de BUTTIKER (6). En Afrique, les espèces du genre Arcyophora, appartenant aux noctuidés sont les plus fortement représentées. Depuis

(*) Cette étude a été réalisée grâce à l'appui financier du Fonds National Suisse de la Recherche Scientifique (requête no 3.503.71).

(**) Institut de Bactériologie Vétérinaire, Université de Berne, CH-3001 Berne, Suisse.

(***) Ciba-Geigy S.A., CH-4002 Bâle, Suisse. longtemps on a soupçonné que ces papillons pourraient jouer le rôle de vecteur de maladies infectieuses chez les mammifères (tableau I), toutefois sans pouvoir préciser les conditions exactes de cette transmission.

Un fait est pourtant frappant : ces papillons sont observés plus fréquemment sur des animaux souffrant d'affections oculaires, où la sécrétion lacrymale abondante offre une source de nourriture très riche. Ainsi chez les bovins et ovins atteints de kératoconjonctivite, maladie très répandue dans les pays tropicaux, il n'est pas rare d'observer ce parasitisme dans de nombreux pays d'Afrique et d'Asie. Selon les données de la FAO, de la WHO et de l'Organisation internationale des épizooties (11), la kératoconjonctivite épidémique est répandue dans beaucoup de pays. Parmi les pays d'Afrique occidentale, la maladie a été observée sur les bovins, ovins et autres mammifères en Côte d'Ivoire, au Mali et en Haute-Volta. Il est actuellement établi que la kératoconjonctivite 


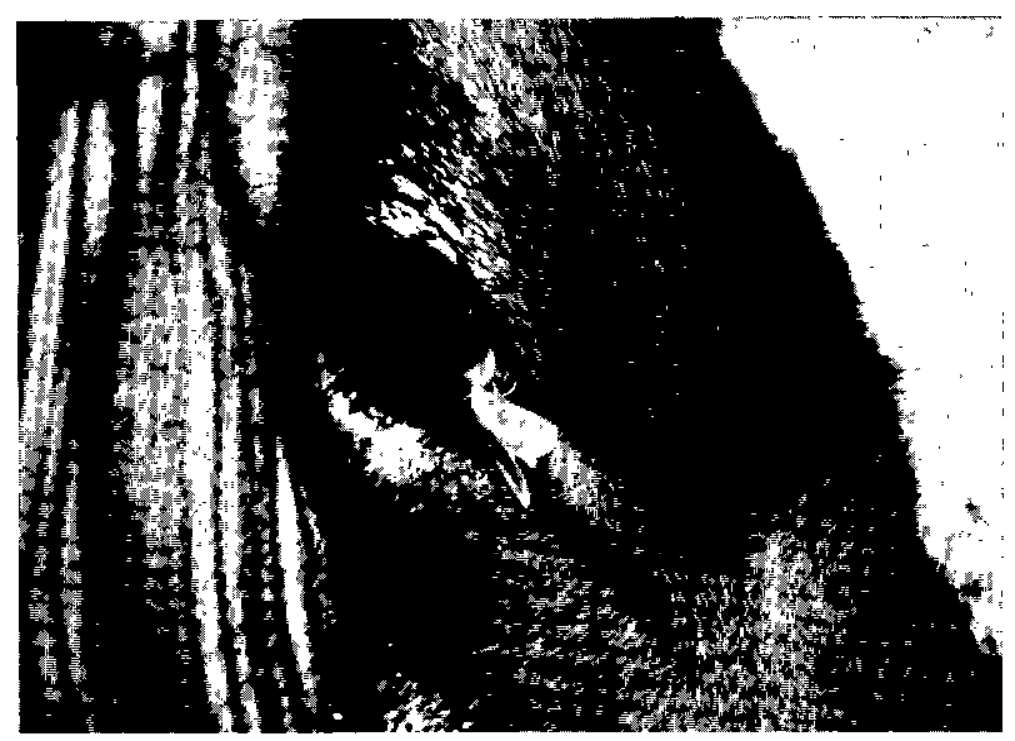

Eil de bovin parasité par un lépidoptère ophtalmotrope (Arcyophora patricula).

TABLEAU I

Références bibliographiques sur le rôle des lépidoptères ophtalmotropes comme vecteurs possibles dans la transmission de maladies infectieuses chez les mammifères (ordre chronologique)

\begin{tabular}{|c|c|c|c|}
\hline Maladie transmise suspecte & Pays ou région & Auteur et année & Réf \\
\hline Ophthalmia in cattle & Rhodésie & Jack R. W., 1915 & (14) \\
\hline African horse sickness & Tanganyika & $\begin{array}{l}\text { Marshall R. et al., } \\
1915\end{array}$ & (14) \\
\hline Temporary blindness & Argentine & $\begin{array}{l}\text { Shannon R.C., } \\
1928\end{array}$ & $(17)$ \\
\hline Ophthalmia & Afrique du Sud & Brain C. K., 1929 & (3) \\
\hline $\begin{array}{l}\text { Some forms of ophthalmia; «... virus diseases } \\
\text { which are carried from game to cattle and } \\
\text { amongst cattle }\end{array}$ & Soudan & Reid E., 1954 & (16) \\
\hline $\begin{array}{l}\text { Infectious ophthalmia; infectious kerato- } \\
\text { conjunctivitis }\end{array}$ & Afrique méridionale & $\begin{array}{l}\text { Henning M. H., } \\
1956\end{array}$ & (13) \\
\hline Infectious ophthalmia & Afrique du Sud & Du Toit R., 1958 & $(10)$ \\
\hline Contagious bovine diseases & Cambodge & Büttiker W., 1959 & (4) \\
\hline $\begin{array}{l}\text { Bovine infectious keratitis, probably transmitted } \\
\text { by a nocturnal moth }\end{array}$ & Afrique orientale & $\begin{array}{l}\text { Guilbride et al., } \\
1959\end{array}$ & $(12)$ \\
\hline Rinderpest, foot-and-mouth disease & 一 & Buttiker W., 1960 & $(15)$ \\
\hline Ophthalmia in cattle & Rhodésie & $\begin{array}{l}\text { Büttiker W., } 1964 \\
\text { Whellan J. }\end{array}$ & $(17)$ \\
\hline $\begin{array}{l}\text { Transmission of pathogens into the conjunctival } \\
\text { sac, on to the cornea, or into the living tissue of } \\
\text { the eyelids }\end{array}$ & Asie sud-est & $\begin{array}{l}\text { Bänziger H., } 1969 \\
\text { Büttiker W., } 1969\end{array}$ & (2) \\
\hline $\begin{array}{l}\text { Microorganismes pathogènes } \\
\text { (y compris spirochètes) }\end{array}$ & & Bänziger H., 1972 & (1) \\
\hline
\end{tabular}


du bovin est une maladie infectieuse dont l'étiologie peut être multiple et qui est favorisée par l'action de facteurs prédisposants (rayons ultraviolets, poussière etc.) (18). Le rôle joué par les insectes a particulièrement retenu l'attention des chercheurs [lit. citée par WILCOX (18)]. On a pensé que les insectes pourraient d'une part exercer une action traumatique sur la cornée et par là favoriser l'infection, ou d'autre part être impliqués directement dans la transmission de l'agent infectieux en qualité de véhicules passifs.

Des recherches avec Moraxella bovis ont confirmé cette dernière hypothèse puisque cet agent a pu être mis en évidence sur les ailes et les pattes de mouches se nourrissant de sécrétion lacrymale d'animaux malades [STEVE and LILLEY cit. par WILCOX (18)]. Par contre Moraxella bovis ingéré par l'insecte est rapidement détruit dans l'appareil digestif, excluant un rôle possible de vecteur actif.

Dans le même ordre d'idée les effets du parasitisme des lépidoptères ophtalmotropes sont fort mal connus. Lors d'une expédition dans le Nord de la Côte d'Ivoire, secteur Korhogo, nous avons tenté de définir le rôle joué par les lépidoptères ophtalmotropes dans la transmission de la kératoconjonctivite du bovin. Dans deux premiers travaux $(8,15)$ nous avons rapporté les résultats entomologiques et déterminé la flore microbienne de cas de kératoconjonctivite chez les bovins en mettant l'accent sur l'isolement de mycoplasmes. Au cours de la présente étude, nous avons examiné les papillons surpris sur ces mêmes animaux, en mettant l'accent également sur l'isolement de mycoplasmes. Nous avons examiné bactériologiquement le proboscis, l'intestin moyen et les excréments de certains noctuidés, une analyse étayée par des observations histologiques de l'intestin. Ces études microbiologiques et entomologiques ont été complétées par des recherches histologiques du système digestif de ces lépidoptères en comparaison avec d'autres groupes de papillons et insectes (9). Quelques résultats seront utilisés dans la présente publication. Il s'agit, vu notre très court séjour en Côte d'Ivoire, d'une étude essentiellement informative, devant fournir les bases d'une enquête plus systématique.

\section{MATERIEL ET METHODES}

Nous avons conduit notre recherche dans 5 troupeaux de village dans un rayon d'environ $30 \mathrm{~km}$ autour de Korhogo. Il s'agissait de bêtes de la race N'dama, généralement métissée avec la race locale Baoulé et dans certains cas avec du Zébu.

Pendant le jour, nous avons visité les troupeaux, repéré les animaux malades auxquels nous avons prélevé des échantillons de la conjonctive pour une analyse bactériologique. Ces animaux ont été ensuite marqués afin de pouvoir être identifiés le soir. La nuit venue, nous avons tenté de surprendre des lépidoptères ophtalmotropes parasitant ces bêtes marquées. C'est alors qu'à l'aide d'un filet nous avons pu capturer précisément ces papillons.

Sitôt arrivés au laboratoire, nous avons procédé à l'analyse bactériologique de ces papillons.

$\mathrm{Au}$ total, nous avons examiné 11 papillons dont l'espèce a été déterminée selon les critères décrits précédemment (15).

Le proboscis a été sectionné à l'aide d'instruments stériles, puis certains spécimens étalés sur une gélose au sang de mouton et systématiquement immergés dans un milieu adéquat pour l'isolement de mycoplasmes.

Après dissection dans des conditions aseptiques, nous avons prélevé avec une anse le contenu stomacal que nous avons ensemencé sur une gélose au sang de mouton et dans le milieu pour mycoplasmes.

Dans certains cas, nous avons eu l'occasion de mettre également en culture des substances excrétées (excréments clairs et opaques) fraîchement libérées. La description des milieux de cultures, les modalités d'ensemencement et les méthodes de différenciation sont citées dans notre étude précédente (15).

\section{ASPECTS ET TECHNIQUES HISTOLOGIQUES}

Les travaux histologiques ont été commencés avec du matériel prélevé dans les années 19631968 en Asie et ont été poursuivis en 19701974 avec des insectes appartenant aux différentes espèces et de différents groupes se nour- 
rissant de sécrétion lacrymale, de pus, de sang, de nectar, etc. Le but de ces recherches était d'étudier comparativement l'anatomie interne du tube digestif, la présence éventuelle de symbiontes et autres germes, et surtout la nature de la nourriture digérée. Les détails des techniques et résultats seront publiés prochainement (9).

\section{RESULTATS}

La bactériologie de 6 papillons surpris sur 6 vaches de 3 troupeaux est résumée dans le tableau II. Nous avons, dans ce tableau, cité à titre comparatif les différentes bactéries isolées sur plusieurs animaux du troupeau et la bactériologie de l'animal parasité.

Pour différentes raisons d'ordre technique, nous n'avons malheureusement pas pu examiner tous les lépidoptères selon les mêmes cri- tères d'examen. Ainsi, seuls 2 papillons présentent une imago complète (vache 641 et vache 645), alors que pour les autres seul l'estomac et au moins un proboscis ont pu être mis en culture.

Dans la même optique et ceci d'une manière plus systématique, le tableau III nous rend compte des résultats de la recherche de mycoplasmes sur 11 papillons surpris sur des bêtes de 5 troupeaux. Ici, également, l'isolement de Mycoplasma bovoculi, dans le troupeau en question et sur l'animal parasité, est mis en regard des résultats obtenus sur les papillons incriminés. Chez 3 bêtes infectées par Mycoplasma bovoculi (vaches 641 et 648 à Pinion, vache $n^{\circ} 2$ à Niofon) nous avons surpris un noctuidé, mais aucun mycoplasme n'a pu être isolé de ces papillons, pas plus d'ailleurs de tous les autres exemplaires examinés et ceci malgré le nombre élevé d'échantillons (24 cultures).

TABL. $N^{0}$ II - Bactériologie de lêpidoptères surpris sur des yeux de bovins, en comparaison avec la bactériologie des yeux de ces bovins et celle d'autres bêtes du troupeau.

\begin{tabular}{|c|c|c|}
\hline $\begin{array}{l}\text { Traupeau } \\
\text { (bactériologie) }\end{array}$ & $\begin{array}{l}\text { Vache } \\
\text { (bactêriologie) }\end{array}$ & $\begin{array}{l}\text { Lépidoptères ophtalmotropes } \\
\text { (bactériologie) }\end{array}$ \\
\hline \multirow[t]{3}{*}{$\begin{array}{l}\text { Pinion } \\
\text { (Moraxe ila nonizque- } \\
\text { faciens, Staphylocoques, } \\
\text { Bacilles) }\end{array}$} & $\mathrm{N}^{0} 641++$ flore mixte & 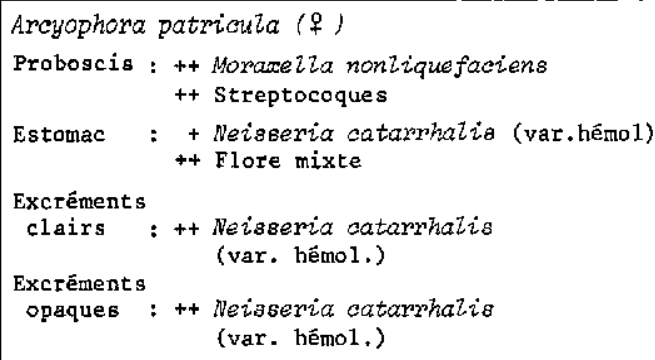 \\
\hline & $\begin{array}{c}\mathrm{N}^{0} 645+\text { Staphylocoques } \\
\text { The Zazia sp. }\end{array}$ & 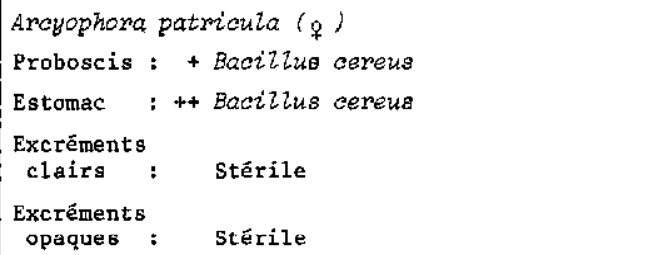 \\
\hline & $N^{*} 648+$ Bacilles & $\begin{array}{l}\text { Arcyophora patricula (q) } \\
\text { Proboscis: + staphylocoques } \\
\text { Estomac : + Klebsiezla aerogenes }\end{array}$ \\
\hline \multirow{2}{*}{$\begin{array}{l}\text { Kasombarga } \\
\text { (Moraxelia bovis, } \\
\text { Neisseria catarrhalis } \\
\text { Klebsiella aerogenes, } \\
\text { Entërocoques, Bacillus } \\
\text { cereus, Staphylocoques) }\end{array}$} & 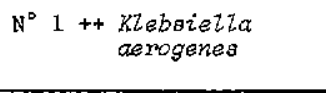 & $\begin{array}{l}\text { Arcyophora patricula ( }) \\
\text { Estomac +++ Bacilizu cereus }\end{array}$ \\
\hline & $\begin{aligned} \mathrm{N}^{\circ 2} & + \text { Moraxelta bovis } \\
& + \text { Staphylocoques }\end{aligned}$ & $\begin{array}{l}\text { Arcyophora patricula (Ъ) } \\
\text { Estomac : +++ Bacilius cereus }\end{array}$ \\
\hline $\begin{array}{l}\text { Lofiné } \\
\text { (Neisseria catarnhalis } \\
\text { var, hëmol. } \\
\text { Mima polzmorpha, } \\
\text { Aeromonas, Bacilles) }\end{array}$ & $\mathrm{N}^{*} 1++$ Aeromonas & $\begin{array}{l}\text { Arcyophora patricula ( } q \text { ) } \\
\text { Estomac : ++ Aeromonas sp }\end{array}$ \\
\hline
\end{tabular}


TABL. N"III-Recherche de mycoplasmes chez 11 papillons surpris sur des vacheo N'Dama métisses de différents troupeaux et souffrant de kératoconjonctivite.

\begin{tabular}{|c|c|c|c|}
\hline Troupeau & $\begin{array}{l}\text { Isolement de } M . \text { bovoculi } \\
\text { dans le troupeau (animal) }\end{array}$ & $\begin{array}{l}\text { Lëpidoptères ophtalmotropes } \\
\text { examinês (animal parasité) }\end{array}$ & $\begin{array}{c}\text { Isolement de mycoplasmes } \\
\text { lếpidoptères }\end{array}$ \\
\hline \multirow[t]{3}{*}{ Pinion } & \multirow[t]{3}{*}{ positif (yaches $n^{*} 641$, } & $\begin{array}{l}\text { Arcyophora patriaula (q) } \\
\text { P, E (vache 641) }\end{array}$ & $2 \times$ négatif \\
\hline & & $\begin{array}{l}\text { Arcyophora patricula ( }) \\
\text { P, E Excr. clairs, Excr. } \\
\text { opaques (vache } 645 \text { ) }\end{array}$ & $4 \mathrm{x}$ négatif \\
\hline & & $\begin{array}{l}\text { Arcyophora patricula }(q) \\
\text { P, E (vache } 648 \text { ) }\end{array}$ & $2 \times$ négatif \\
\hline \multirow[t]{2}{*}{ Karakoro } & \multirow[t]{2}{*}{ positif (veau $n^{*} 4$ ) } & $\begin{array}{l}\text { Arcyophora patricula (o) } \\
\text { P, E (vache 1) }\end{array}$ & $2 \times$ négatif \\
\hline & & $\begin{array}{l}\text { Aroyophora patricula (o) } \\
\text { P, E (vache 2) }\end{array}$ & $2 \times$ négatif \\
\hline \multirow[t]{2}{*}{ Niofon } & \multirow[t]{2}{*}{ positif (vache $\left.n^{*} 2\right)$} & $\begin{array}{l}\text { Arcyophora patricula ( } q) \\
\text { P, E (vache 1) }\end{array}$ & $2 x$ négatif \\
\hline & & $\begin{array}{l}\text { Arcyophora patricula (d) } \\
\text { P, E (vache 2) }\end{array}$ & $2 \times$ négatif \\
\hline \multirow[t]{2}{*}{ Kasombarga } & \multirow[t]{2}{*}{ positif (veau $n^{\circ} 4$ ) } & $\begin{array}{l}\text { Arcyophora patricula (o) } \\
\text { P, E (vache 1) }\end{array}$ & $2 \times$ négatif \\
\hline & & $\begin{array}{l}\text { Arayophora patricula (0) } \\
\text { P, E (vache 2) }\end{array}$ & $2 \times$ négatif \\
\hline \multirow[t]{2}{*}{ Lofiné } & \multirow[t]{2}{*}{ nëgatif } & $\begin{array}{l}\text { Arcyophora patricula (\%) } \\
\text { P, E (vache 1) }\end{array}$ & $2 \times$ négatif \\
\hline & & $\begin{array}{l}\text { Arcyophora longivalvie ( }) \\
\mathrm{P}, \mathrm{E} \text { (vache 2) }\end{array}$ & $2 x$ négatif \\
\hline
\end{tabular}

$(P=$ Proboscis; E - Estomac; Excr. = Excréments $)$

En ce qui concerne l'examen histologique de l'intestin moyen des lépidoptères surpris après l'acte de nutrition, il est important de savoir que ces noctuidés se nourrissent essentiellement de sécrétion lacrymale et de sécrétion purulente de mammifères. Les exemplaires inclus dans cette étude montrent en effet que leur contenu intestinal se compose principalement des mêmes cellules que l'on trouve dans la sécrétion lacry- male des bovins affectés de kératoconjonctivite. Il s'agit de grosses cellules épithéliales semblables à celles de la conjonctive et de cellules leucocytaires. On note de plus l'absence d'hématies et de pollen, alors que les bactéries sont en général peu nombreuses.

La numération différentielle des cellules $\mathrm{du}$ contenu intestinal est donnée pour 2 espèces de lépidoptères dans le tableau IV.

TABL. N*IV-Différenciation quanticative des cellules du contenu de 1 'intestin moyen d'Arcyophora patricula et Areyophora longivalvio. (9)

\begin{tabular}{|c|c|c|c|}
\hline \multirow{2}{*}{ Lépidoptère } & \multirow{2}{*}{$\begin{array}{c}\text { Cellules } \\
\text { épithêliales }\end{array}$} & \multicolumn{2}{|c|}{ Leucocytes } \\
\hline & & $\begin{array}{l}\text { neutrophiles à } \\
\text { noyau segmenté }\end{array}$ & Lymphocytes \\
\hline $\begin{array}{l}\text { Areyophora patricula } \\
\text { (n = 9) } \\
\text { Arcyophora longivalvis } \\
(n=3)\end{array}$ & $\begin{array}{l}35,3 \text { p.100 } \\
30,1 \text { p. } 100\end{array}$ & $\begin{array}{l}57,0 \text { p.100 } \\
60,1 \text { p.100 }\end{array}$ & $\begin{array}{l}7,7 \text { p. } 100 \\
9,8 \text { p. } 100\end{array}$ \\
\hline
\end{tabular}

(n = nombre d'exemplaires) 


\section{DISCUSSION}

D'après les observations faites en Afrique occidentale, il est indéniable que les lépidoptères ophtalmotropes parasitent de préférence les yeux des bovins sujets à des sécrétions lacrymales anormales. Ces papillons peuvent ainsi se contaminer avec du matériel infectieux et le cas échéant, lors du parasitisme d'un autre animal, transmettre des germes qui peuvent être à l'origine d'une nouvelle infection ou aggraver une infection déjà présente. Il est en effet reconnu qu'un papillon fréquente généralement plus d'un animal au cours de son acte de nutrition.

Nous avons observé que les irritations oculaires sont fréquentes dans les troupeaux visités dans le nord de la Côte d'Ivoire, avec enflement des paupières, photophobie et lacrymation abondante. Un certain pourcentage de ces animaux montre des lésions plus ou moins avancées de kératoconjonctivite, ce qui, vu la flore bactérienne isolée, laisse supposer une origine infectieuse. Les lépidoptères ophtalmotropes peuvent à notre sens être véhicules d'agents infectieux, ceci d'autant plus que leur proboscis, qui agit directement sur la conjonctive, peut se contaminer fortement et que, par sa morphologie en dents de scie et par ses vibrations intenses, il y ajoute encore un élément traumatique local. Ceci, à notre avis, attribue aux lépidoptères ophtalmotropes un rôle certainement plus actif que celui des différentes espèces de mouches (Musca domestica, $M$. fasciata, $M$. sorbens, Stomoxys spp.) dans la transmission possible d'agents infectieux.

Nos résultats, quoique très partiels, permettent de soutenir une telle hypothèse. Nous retrouvons en effet sur le proboscis de ces papillons une flore bactérienne que nous isolons communément à partir des conjonctives de bovins affectés de kératoconjonctivite dont un représentant classique est Moraxella nonliquefaciens $(15,18)$. Si nous ne retrouvons pas sur le proboscis la même flore que sur la conjonctive de l'animal parasité, il faut remarquer que ces germes ont été généralement isolés sur d'autres bêtes du même troupeau. Il faut tenir compte du fait que ces papillons. ont été capturés peu après s'être posé et que par conséquent ils n'ont pas eu l'occasion d'avoir un contact prolongé. Donc, selon les circonstances, la flore isolée sur le proboscis est représentative d'un autre animal.
Nous nous sommes intéressés au sort de ces germes lorsqu'ils passent par le tractus gastro-intestinal.

Nos résultats concemant la bactériologie du contenu stomacal montrent que nous retrouvons ici encore des germes connus pour avoir été isolés sur la conjonctive de bovins, tels Neisseria catarrhalis var. hémolytique, Klebsiella aerogenes et Aeromonas. Nous avons été pourtant frappés de rencontrer fréquemment un germe que nous avons morphologiquement identifié comme Bacillus cereus, que l'on retrouve certes sur les yeux de bovins, mais que très sporadiquement. Peut-être que la ténacité de ce germe lui permet de survivre dans ce milieu au détriment d'autres bactéries.

Un autre fait curieux est l'isolement pratiquement en culture pure dans les excréments de Neisseria catarrhalis var. hémolytique. En effet, lors du processus digestif, l'aliment est filtré d'une manière très active au niveau de l'intestin et les résidus sont ensuite excrétés sous forme de gouttes opaques et claires. Il serait logique que ces excréments soient en réalité stériles, comme les résultats d'un papillon nous l'ont montré. Nous ne connaissons pas l'efficacité de cette filtration, mais en ce qui concerne le genre Neisseria, nous pouvons affirmer que ce sont des bactéries relativement petites, ce qui pourrait situer la perméabilité de ce système pour des particules de l'ordre du micron.

L'examen histologique de l'intestin moyen et de son contenu montre qu'en général les bactéries y sont peu représentées. Par contre, on constate une analogie frappante entre l'image cellulaire du contenu intestinal et celle de la sécrétion lacrymale purulente de l'hôte parasité. Ce qui d'une part apporte une confirmation que les lépidoptères ophtalmotropes se nourrissent activement de sécrétion lacrymale de mammifères et d'autre part qu'ils se nourrissent de préférence sur un hôte ayant une abondante sécrétion comme c'est le cas lors d'affections oculaires. Ce phénomène est mis particulièrement en évidence par BANZIGER (1) qui trouve des leucocytes dans 87 p. 100, des cellules épithéliales dans 85 p. 100 des contenus intestinaux examinés. Ces observations soulignent une fois encore la sélectivité de l'hôte et le rôle possible des lépidoptères dans la propagation d'agents infectieux d'un animal à l'autre. 
La recherche de mycoplasmes qui étaient pourtant fortement représentés par Mycoplasma bovoculi soit dans le troupeau, soit en partie sur les bovins parasités, s'est soldée par un échec. L'explication la plus plausible est que la technique d'isolement de ces organismes est très difficile, et qu'une culture en milieu synthétique n'est possible qu'avec un inoculum contenant suffisamment de germes de départ. Cette condition n'est certainement pas remplie avec l'immersion dans un bouillon de culture du minuscule proboscis. Nous attendions plus de la culture du contenu stomacal ou des excréments. Il est pensable dans ce cas que la faible ténacité des mycoplasmes n'ait pas résisté aux conditions du milieu intestinal. Ainsi cet échec ne permet pas d'écarter l'hypothèse que ces mycoplasmes puissent être malgrê tout véhiculés par les noctuidés.

Ainsi le rôle épidémiologique des lépidoptères ophtalmotropes dans la transmission possible de la kératoconjonctivite infectieuse du bovin doit être pris sérieusement en considération. Le proboscis joue certainement un rôle central, du moins dans la transmission passive. Par contre, le rôle joué par l'appareil digestif ne nous est pas clair. D'une part nous savons que ces papillons ne sont pas doués de la propriété de régurgiter leur contenu stomacal et d'autre part les excréments sont généralement expulsés pendant et après l'acte de succion partiellement après le retour du papillon dans son habitat. II est enfin improbable que ces bactéries accomplissent dans l'insecte un cycle de reproduction, ce qui n'attribuerait à ces papillons qu'un, rôle de véhicules passifs d'agents infectieux. Il serait toutefois désirable que ces investigations incomplètes puissent être menées plus systématiquement. Notre dessein n'étant ici que de soulever un problème très méconnu.

\section{Remerciements}

Nous tenons en premier lieu à remercier Dr P. LINDLEY, FAO, Korhogo, M. E. TIDORI et Dr J. A. AJUZIOGU, Centre d'élevage, Bouaké; M. R. CADOT et Dr J. MATHON, I.E.M.V.T., Minankro et leurs assistants qui tous nous ont généreusement aidés pendant les travaux faits en plein champs et au laboratoire.

\section{SUMMARY}

Observations on cattle infectious keratoconjunctivitis in Ivory Coast. II. Study of the ophthalmotropic Iepidoptera as vector

In a first study the role played by the eye-frequenting lepidoptera in the transmission of the keratoconjunctivitis epidemica in cattle has been investigated. Moraxella nonliquefaciens and other germs have been isolated on the proboscis; in the mid gut Neisseria catarrhalis var. haemobytica, Bacillus cereus, Aeromonas, Klebsiella aerogenes and a mixed flora was found. The excrements were either infested by Neisseria catarrhalis var. haemolytica or they were sterile. We were unable to isolate any mycoplasmas from proboscis, mid gut or from the excrements of the eleven specimens investigated. There are strong indications that the ophthalmotropic lepidoptera are mechanical vectors of eye diseases such as keratoconjunctivitis.

\section{RESUMEN}

Observaciones sobre la queratoconjuntivitis infecciosa del bovino en Costa de Marfil.

II. Estudio sobre el papel vector de los lepidópteros oftalmotropos

Los autores trataron de determinar el papel desempeñado por los lepidópteros oftalmotropos en la trasmisión de la queratoconjuntivitis del bovino.

Según el aislamiento sobre el proboscis de ciertos germenes, en particular Moraxella nonliquefaciens, en el intestino y los excrementos de Klebsiella aerogenes y de Aeromonas sp., se puede suponer que las mariposas trasmitan tales bacterias de un animal al otro.

No fué posible aislar ningún micoplasmo del proboscis, del intestino o de los excrementos de 11 mariposas observadas. 


\section{BIBLIOGRAPHE}

1. BANZIGER (H.). Biologie der lacriphagen Lepidopteren in Thailand. Rev. Suisse Zool, 1972, 79 (4) : 1381-2469.

2. BANZIGER (H.), BƯTTIKER (W.). Records of eye-frequenting Lepidoptera from Man. J. med. Ent., 1969, 6 (1) : 53-58.

3. BRAIN (C. K.). Insect pests and their control in S. Africa. Le Cap, C.N.A., 1929

4 BUTTTIKER (W.). Observations on feeding habits of adult Westermanniinae (Lepid., Noctuidae) in Cambodia. Acta Trop., 1959, 16 (4) : 356-361.

5. BUTTIKER (W.). Biological and morphological notes on the fruitpiercing and eye-frequenting moths. in: 11th Int. Congr. Entomol., Vienne, 1962, $2: 10-15$.

6. BUTTIKER (W.). Vorläufjge Beobachtungen an augenbesuchenden Schmetterlingen in der Elfenbeinküste. Rev. Suisse Zool., 1973, 80 (1) : 1-43.

7. BUTTIKER (W.), WHELLAN (J.A.). Records of eye-frequenting moths from Rhodesia. Rhod. agric. J., 1966, 63 (2) : 24-27.

8. BUTTIKER (W.), NICOLET (J.). Observations complémentaires sur les lépidoptères ophtalmotropes en Afrique occidentale. Rev. Elev. Méd. vét. Pays trop., (A paraître).

9. BUTTIKER (W.), KRINE (G.), SCHOPFER (T.). Comparative investigations on the digestive tract of Lepidoptera with special reference to eyefrequenting moths. (en préparation).

10. DU TOIT (R.). Veterinary Research Station, Onderstepoort (Pretoria). Communication personnelle.

11. FAO/WHO/OIE. Animal Health Yearbook 1972. Rome, FAO, 1973.

12. GUILBRIDE (P), BARBER (L,), KALIKWANI (A.). Bovine infectious keratitis suspected mothborne outbreak in Uganda. Bull, epiz. Dis. Afr., 1959, $7: 149-154$.

13. HENNING (M. H.). Animal Diseases in South. Africa. Le Cap, CNA, 1956.

14. MARSHALL (G.A.K.), JACK (R. W.). A noctuid moth feeding on the moisture from the eyes of mules. Trans. r. ent. Soc. London, 1915, 117-119.

15. NICOLET (J.), BUTTIKER (W.). Observations sur la kératoconjonctivite infectieuse du bovin en Côte d'Ivoire. 1. Aspects microbiologiques. Rev. Elev. Méd. vét. Pays trop., 1975, 28 (2): 11.5-124.

16. REID (E.T.M.). Observations on feeding habits of adult Arcyophora. Proc. R. ent. Soc. Lond. (B), 1954, 23 (11-12) : 200-204.

17. SHANNON (R. C.). Zoophilous moths. Science, London, 1928, 68 (1767) : 461-462.

18. WILCOX (G. E.). Infectious bovine keratoconjunctivitis : a Review. Vet. Bull., 1968, 38 (6) : 349-360. 